\title{
An outline of the basic theory of accident science based on the safety science methodology in risk process
}

Tongyuan $\mathrm{Luo}^{a} *$, Chao $\mathrm{Wu}^{b, c}$

a School of Electronic Engineering, Xi'an Shiyou University, Xi'an 710065, China

${ }^{b}$ School of Resources and Safety Engineering, Central South University, No.932 South Lushan Road, Changsha, Hunan 410083 P.R. China

${ }^{c}$ Safety \& Security Theory Innovation and Promotion Center (STIPC), Central South University, No.932 South Lushan Road, Changsha, Hunan 410083 P.R. China

* Frist and corresponding author. E-mail: tongyuan.luo.china@hotmail.com

\begin{abstract}
In order to establish a new discipline specializing in accident science from the perspective of safety science. Under the guidance of the current research theories and methods of safety science, combined with the research paradigm of humanities and social medicine, this paper puts forward new viewpoints, new theories and new models about accident research. First of all, through literature retrieval, this paper analyzes the relevant research results of accidents at home and abroad, and expounds the existing problems and the basic trend of accident science research. Secondly, it puts forward eight kinds of attribute relations of the accident, and makes clear the characteristics and connotation of the accident. In the study of accident types, a hierarchical classification model based on accident cognition is created for the first time. It also points out the logical relevance of five levels of accident science research and the realistic relevance of three levels. At the same time, according to the thought of science of science, this paper puts forward a new definition of safety under the thinking of accident science and other basic concepts related to safety science, and explains the connotation. In addition, it creates and constructs the basic concept of accident science, establishes the conceptual model of accident science, and points out the "3-4-5" model of accident science research and its connotation. Thirdly, draw lessons from the interdisciplinary paradigm to study the relevant theoretical basis and discipline classification relationship of accident science, and construct the tree of accident science. Finally, the research contents of three main aspects of accident science are summarized. The results show that the research results in this paper not only play a fundamental role in the basic construction of accident science, but also further enrich and perfect the discipline system of safety science, which has a certain theoretical significance.
\end{abstract}

Keywords: Accident; Accident connotation; Accident science; New definition of safety; Conceptual model of accident science; Discipline basic construction

\section{Introduction}

Today, the world is in the wave of great development, adjustment and change of industry and production. In various fields of the world, human beings are faced with the risks and challenges brought by changes all the time, in which all kinds of accidents and disasters emerge one after another. Aside from the death brought by war, perhaps the casualties have always accompanied the human beings and never left. The casualties in times of war were heavy, and the injuries in times of peace were also shocking. Since ancient times, human beings have begun to fight against accidents, for which hundreds of millions of people around the world have given their lives. Accidents are like the sword of Damocles hanging over their heads, which will lead to death without warning. The history of human society is accompanied by accidents, so for thousands of years, especially since modern times, with the advent of three revolutions, accidents seem to occur frequently people's lives, especially several major accidents that shocked the world so that people feel afraid when talk about them (LUO and WU, 2018; ZHANG, 2013). Accidents can cause as much pain as diseases, which makes people attempt to avoid it.

Regarding accident-related issues, foreign scholars have conducted research for a long time: Underwood et al. (Underwood and Waterson, 2013) believed that the accident was the result of the undesired and uncontrollable correlation among system components (i.e. safety information), and pointed out that one of the reasons for the disconnection between the research and practice of accident cause theory is to ignore the safety relationship of system's components; Abbas Zaranezhad et al. (Zaranezhad et al., 2019) proposed a causal model for oil refinery maintenance-related accidents, combined with artificial neural networks, fuzzy systems and meta-heuristic algorithms to propose the best model for early accident prediction. The main factors that affect the occurrence of accidents are classified into external factors, internal factors, executive factors, behavioral factors, situational factors and work factors, and the accident type prediction, consequence type prediction and population density 
prediction are realized. Mikolaj Oettingen (Oettingen, 2020) applied the criticality analysis method to analyze the undesired critical state of the accident, and designed the Monte Carlo continuous capacity analysis method by referring to the supercritical concept in the physical field, which realized the critical determination of the accident status and provided a new idea for the occurrence of the accident. Ali J. Ghandourd et al. (Ghandour et al., 2020) developed an online platform for road traffic accidents in Lebanon to extract allometric growth scales for studying accidents, including data on different parameters, such as area size, population size of each area, and road network length, etc., providing a time clustering model and behavior patterns for traffic accidents; Hasan H. et al. (Joni et al., 2020) studied the traffic accidents in Iraq over the past decade, established a data set of accident statistics, and pointed out that the rapid increase in the accident rate due to the lack of security and safety precautionary measures; Amir Bahador Parsa et al. (Parsa et al., 2020) used machine learning technology based on extreme gradient enhancement to detect the occurrence of accidents and revealed the changes of vehicle speed before and after traffic accidents through the data processing of traffic, network, population, land use and weather characteristics. In addition, Amir used the joint interpretation of the average daily traffic flow at the upstream location and the speed after the accident / non-accident time to calculate the time and location before and after the accident through the model. D.W. Hummel et al. (Hummel et al., 2020) studied the prediction model of limiting accident of small modular nuclear reactor, designed the appropriate emergency planning zone for the radiation dose caused by the leakage of nuclear accident, and calculated the minimum evacuation criteria to prevent accident risk by using atmospheric dispersion and dose consequence. Thus, simulation reference for accident prevention and consequence emergency was provided. Karen Landay et al. (Landay et al., 2020) investigated the relationship between the personality characteristics of professional truck drivers and the accident according to the company's accident records over the years. Analysis showed that people who were more understanding were less likely to have an accident, while people who were more anxious, more guilty, more expressive, and more adventurous were more likely to have an accident. Bernard Youkhana et al. (Youkhana et al., 2019) analyzed the technical and clinical data of patients with facial injuries caused by car accidents, and investigated factors such as sitting posture, sex, age, accident time, use of seat belts, deployment of front airbags, direction of impact, collision speed at that time, occurrence and location of facial injuries, and so on. It was concluded that the primary factor causing injury in a collision accident is the high speed at the time of collision. And car safety measures reduced injury to people in the event of an accident. Ludwig Benner Jr. (Benner, 2019) studied the basic issues such as the definition of accident and investigation, the purpose of accident investigation, and the cause of the accident, which showed the diversity of thinking in this kind of accident investigation, and explained the reasons for the existence of so many methods of investigation and analysis. These studies guided people how to use accident data for analysis and investigation. Marislei N. and Fabiana F.R. (Nishijima and Rocha, 2020) researched the accident consequences of tailing dam failing and pointed out that the accident losses included: deaths and injuries, economic losses, environmental pollution and health problems associated to heavy metals in the water. This study found evidence that the dam accidents had a positive and statistically significant impact on dengue indicators. Nadia K.N. (Naqvi et al., 2020) believed that improvements in vehicle and road design, medical technology and care, and driver education and training have decreased the incidence of traffic accidents and several evidences showed that fuel price changes also had a major impact on road traffic accidents through other mediating factors. For example, less car travel and more fuel-efficient driving like speed reduction on high-speed roads could reduce driver exposure. Furtherly, it was proved that the high fuel price increased road traffic accidents. Ryan W.A. et al. (Allison et al., 2019) provided a methodology and costing structure that facilitates the calculation of true construction accidents costs, used an incidence-based,'ex-post'approach to promote the internalization of true accident costs and positively proposed the improvement of safety programs. A. Fyhri, O. Johansson, T. Bjørnskau (Fyhri et al., 2019) investigated the causes of accidents in electric bicycles and used logistic regression analysis to compare the accident factors of traditional and e-bikes. The results suggested an overall risk increase (all 
accidents) for e-bike users, and the increased risk derives from females having a higher accident risk on e-bikes. Meanwhile, an in-depth analysis of accident causation showed that there was no difference in the factors leading to accidents, except that there was a somewhat higher prevalence of accidents resulting from balance problems with e-bikes. Lina Jonsson et al. (Jonsson et al., 2019) assessed the accident risk for both motorized road traffic and vulnerable road users and thought that protection device, road type, traffic volume of the trains, and number of persons living nearby the level crossing effected the accident probability significantly. Using marginal effect of train traffic on the accident risk derived the marginal cost per train passage caused by level crossing accidents. Madhav S. Aney, Christine Ho studied the impact of the Road Traffic Safety Law on traffic safety. Although the law succeeded in reducing the number of accidents and casualties, the ratio of deaths to accidents and injuries to accidents was on the increase(Aney and Ho, 2019). An increase in the severity of accidents could probably be consistent with a model where all motorists drive more carefully after the reform, but have heterogeneous responses. In other words, the decrease in accident probability is larger for safer than for riskier drivers. Shohel Amin determined that the purpose of travel, lighting conditions, pedestrian crossing with physical interventions, complex road geometry, extreme weather and time severity were the most important factors that cause accidents for elderly drivers (Amin, 2020). By providing accessible routes, economical, reliable and convenient public transportation, timely warning of accidents and changes in road geometry, the accident risk of elderly drivers could be improved. Nicolas Dechy et al. (Dechy et al., 2012) proposed a strong process of convergence, coordination, concentration, best practice, and scientific knowledge sharing, which may improve the accident investigation process and may prevent accidents in the future. The follow-up action of lessons learned from accident investigation should focus on dynamic learning, and accident investigation should be a multi-level learning process with the background of culture, society, system, organization, epistemology and methodology in the social and technological system. Pramod Kumar, Suprakash Gupta, Yuga Raju Gunda put forward a methodology for estimation of human error rate from a retrospective analysis of accident reports using fuzzy mathematical concepts. The proposed approach used accident reports of underground coal mines for assessing the human error rates of essential mining activities, identifying the critical activities and error types. It also suggested some error reduction strategies for devising an intervention to accidents (Kumar et al., 2020). José A. García-Arroyo et al. (García-Arroyo and Osca Segovia, 2020) explored whether differences in language, the cultural distance between countries, and national cultural values could explain the accident rate and its variability in different economic sectors (agriculture, industry, construction and services) and analyzed accident rates of immigrant workers from in Spain. Results showed that speaking a different language and being culturally different did not influence accidents in immigrants, except in agriculture where speak a different language was associated with fewer accidents. Cultural values by themselves, instead of language or cultural distance between countries, were better predictors of the variance of work accidents in immigrants. Hamidreza Mokarami et al. (Mokarami et al., 2019) investigated the relationship between safety culture and unsafe behaviors, and accidents among bus drivers. The results indicated a negative relationship between accidents, and safety culture and drivers' unsafe behaviors. A positive association was also observed between unsafe behaviors and accidents. The unsafe behavior significantly mediated the relationship between safety culture and accidents and strategies for improvement of organizational safety culture could decrease the drivers' unsafe behaviors and subsequently reduce the number of accidents. Zahra Naghavi K. et al. (Naghavi K et al., 2019) believed that preventing confined space accidents requires addressing issues from the organizational to operator level and external influences beyond the organization. Accident investigation and risk analysis provided the basis for accident prevention, revealing that organizational and supervisory factors in the causes of confined space accidents were the main reasons. John C. Mauro et al. (Mauro et al., 2018) believed that there was a power-law relationship between the number of accidents and their severity. This power-law scaling was indicative of workplace accidents being governed by self-organized criticality, suggesting that nearly all workplace accidents have a common underlying cause, 
independent of their severity. Researches supported that suppressing the rate of severe accidents required changing the attitude toward workplace safety in general and creating a culture that values safety. Zobair Ibn Awala and Kazuhiko Hasegawa (Awal and Hasegawa, 2017) revealed that maritime accidents took place in a complex socio-technical context, and it was necessary to discuss logical planning techniques, epidemiological theories, and system control theories to analyze and understand the accidents. From an engineering point of view, accidents might be treated as a system control problem, and accident theories/models had been originated from different disciplines and the theories/models had been evolving with the changes in the society. Sunday A.A. et al. (Adedigba et al., 2016) presented a new non-sequential barrier-based process accident model. The conditional dependencies among accident contributory factors within prevention barriers were modelled using the Bayesian network with various relaxation strategies, and non-sequential failure of prevention (safety) barriers. The modelling of non-linear interactions in the model led to significant improvement of the predicted probability of an accident when compared with that of sequential technique.

Compared with the research on accident-related technology and engineering strategy abroad, domestic scholars also have a lot of research on accident basic theory, and there is a "two-wheel drive" trend of accident engineering and accident basic research in our country. The main literature is divided into two categories: basic research and engineering technology. The basic research literature on accidents mainly includes the followings. Luo Tongyuan and $\mathrm{Wu}$ Chao(Luo and $\mathrm{Wu}, 2019)$ proposed an accident cause theory based on the cognitive model of safety information, which studied the role of safety information in the occurrence of accidents from information dissemination and cognitive processes and explained that the cause mechanism of accidents was the lack/distortion of the dissemination of safety information. Finally, accident prevention measures for safety information flow was raised. Huang Lang et al. constructed the conceptual model of accident chain evolution based on material flow, energy flow and information flow and summarized the strategy framework of accident prevention and control(HUANG et al., 2016). Fu Gui et al. (Fu et al., 2020) clarified the development history of accident causation models over the past 100 years, and believed that 'Organisational factors' would be replaced by more modern 'safety management systems' and people would pay more attention to the role of 'safety culture' in accident prevention. Accident causation models will develop in a linear and systematic way. The current accident causation models consisted mainly of qualitative analysis and quantitative analysis, and would develop in the direction of dynamic analysis, accident prediction, and intelligent comprehensive analysis in the future. Fu Gui et al. (FU et al., 2018) had the comparative analysis on ten accident causation models from three dimensions of the affected objects of accident, the composition of the model and the path of the accident occurrence. The results showed that the accident causation models are getting more and more comprehensive for the cover on the affected objects of accident. The composition of the accident causation models is getting more comprehensive, and there is a trend to make a clear definition of each module in the model to ensure the accuracy of the analysis results. The description of the path of the accident occurrence develops from the chain type to the system network type. Luo Tongyuan and Wu Chao (Tongyuan and WU, 2018) researched the research object of the accident mechanism should be the human body and the substance, put forward the three major points of reason, conditions and inducing factors, analyzed the research object of the accident and the general law of accident development, and defined the cause of the accident. TU Siyu et al. analyzed the causation factors of the malignant human accidents from the individual factors, group factors and external factors and respectively constructed the models of individual, group and comprehensive malignant human accidents from the behavior chain, causation chain and reason chain. The occurrence mechanism models of the malignant human accidents mainly included the model of behavior safety aiming at the individual malignant human accident, the model of behavior safety aiming at the group malignant human accident and the model of behavior safety aiming at the comprehensive malignant human accident. The bad culture was the root cause, the management defects were the essential cause, the emotional factors, psychological factors and control factors were the indirect causes, and the malignant destruction was the 
direct cause (TU et al., 2018). Feng Qingshan believed that the cause of the accident could be analyzed according to the simulation logic relationship such as the withering of the tree or the breakage of the dry branch, and put forward the accident cause theory of "tree growth". The theoretical criterion was more systematic in terms of the definition of the root cause, direct cause, indirect cause, and identification content of the accident, avoiding the omission or deviation of the cause and analyzing the deep-seated causes of the accident(FENG, 2014). Fu GUI researched the division of cause factors at various stages of internal and external causes of the organization in the accident cause "2-4" model of accidents, analyzed the cause factors in each cause module through accidents, and obtained unsafe actions, physical states, and habitual unsafe behavior, safety management system, safety culture, external factors and so on (FU et al., 2017). Zhou Xin-yu and Guo Sheng-yu explored the mechanism of behavioral risk chain for building construction accident based on complex network and behavioral risk chains were extracted. Furthermore, Pajek was used to construct network model and degree and accumulation degree distribution were analyzed to describe the characteristics of the network (ZHOU and GUO, 2019). The results showed that input and output high-degree nodes reflected common direct and indirect behavioral reasons of accidents, respectively. Yu Fan et al recorded some crowded stampede cases for nearly 16 years. Based on the event system theory, and combining with the characteristics of the event analyzes mechanism of accidents, establishing the mechanism figure of stampede events, they put up the prevention and risk evaluation method for the model of events (YU et al., 2016). The stampede cases were happened under the event with strength special time and great influence to the organization. The cause of vehicle traffic accidents was a catastrophe happening in the operation in the state of vehicle and construct a swallowtail catastrophe model for the operation state of passenger vehicles. Then putting forward that the effective method of prevention of traffic accidents was to prevent the operation state of the vehicle system getting across the bifurcation set (HENG et al., 2017). Lin Yongming (LIN, 2019) took into account the characteristic features of the topics, emotions and dynamics in the online reviews of coal mine safety accidents, the LDA model here to be introduced should be said based on related variables at the emotion and the time juncture. The factors that can cause the coal mine safety accidents were also to be introduced into a social model as well as into a dynamic thematic emotion model to identify public opinions automatically. The results showed that the public opinion analysis model proposed can help to describe the accident topics and the dynamic changes of emotions properly that the public were concerned about. It could be found that the themes and the emotional factors might also significantly affect the outbreak of the public opinion, which could further assist the policy makers in studying and judging the developmental trend of the public opinion on the said coal mine accidents. Li Xiangxin discussed mechanism of the multi-hazard coupling effect based on analyzing multi-hazard coupling types. Hazard area model of accumulation and amplification effect was determined through the disaster initial risky index as well as the risk composite index of harm extent for the multi-disaster coupling accidents (LI et al., 2019). Zhang Jianshe et al. (ZHANG et al., 2019a) analyzed the effect pathways of safety accidents on each dimension as well as an empirical test by using structural equation model. The results showed that safety accidents had a significantly direct impact on facilities environment, institutional environment, and relevant party environment, among which the influence on facilities environment and institutional environment was greater than that on the relevant party environment. Secondly, safety accidents had a significantly indirect impact on safety values and behavioral patterns, among which its indirect impact on behavioral patterns was higher than that on safety values. To reduce the impact of safety accidents on organizational culture, the construction enterprise could improve the environment of the construction site, strengthen the establishment of safety systems and improve its reputation et al.

The accident engineering research literature mainly includes the followings. Mingguang Zhang analyzed several domino accidents of storage tank area to establish theoretic models and event tree. Probability distribution of initial accident and four typical accident node escalation models were picked up: fire $\rightarrow$ fire, fire $\rightarrow$ explosion, explosion $\rightarrow$ fire, explosion $\rightarrow$ explosion. Propagation probability model of accident chain was constructed, 
which consists of initial accident probability, accident node escalation probability and target tank failure probability (Zhang et al., 2019b). Jinjia Zhang et al. (Zhang et al., 2020) pointed out that gas explosions, mine water inrushes, and coal dust explosions were the three major types of extraordinarily severe coal mine accidents (ESCMAs) and inadequate implementation of safety measures, deliberate violations and electromechanical equipment faults were the three top causes of ESCMAs. Qing Liu et al. (Liu et al., 2020) concluded that the coal mine accidents data had the characteristics of large fluctuations, small sample size, small value and large numerical nonlinear characteristics. Policy intervention degree (PID), Manufacturing Purchasing Managers' Index (PMI), Producer Price Index for Industrial Products (PPI), Main raw material purchase price index (RMPPI), Employment index (EI) were selected as the auxiliary variables to construct the early warning model to realize the prediction of the coal mine accident. Jihong Chen et al. (Chen et al., 2020) studied the dangerous goods accidents at ports, so as to identify major factors and prevent them and introduced formal concept analysis (FCA). They built a concept lattice model of dangerous goods accidents at China's ports, and reduced the condition attributes to come up with three key attributes of dangerous goods accidents at China's ports: warehousing management, facilities and equipment, goods registration and extract four effective diagnostic rules for dangerous goods accidents at ports. Hua Lingling and Zheng Wei (HUA and ZHENG, 2019) analyzed railway accidents from four aspects, including the act of man, mechanical equipment, environment and management. Based on the correlation coefficient of Pearson, a network of railway accident causation factors was established. The results showed that management factors and human factors are the most likely to cause railway accidents. At the same time, sub-factors such as illegal operation, communication failure, mechanical failure of components had great influence on railway safety operation. Zhang Li-xia et al. (ZHANG et al., 2014) analyzed the relationship between total number of road traffic accident deaths and that of deaths caused by driving age of 1 to 5 years and accidental death distribution. Drunk driving, fatigue driving, speeding and failing to yield have the greatest impacts on road traffic accidents and drivers with driving age less than 3 years had the greatest influence on accidental deaths. Gan Xusheng et al. (GAN et al., 2015) proposed an analysis method based on Human Factor Analysis and Classification System ( HFACS) for midair collision accident. It analyzed the causation of accident according to the thinking from dominant error to latent error, and finally found out the effect of organizational factors on accident. Xiao Zhongming et al. (XIAO et al., 2017) pointed out that the factors that account for the ship grounding should be attributed to the human navigation factors, the ships own construction factors and the environment-affecting factor, and established the Bayesian network model about the ship grounding. They had managed to gain the probability of the influential factors and the maximum causation chain of ship grounding accidents with the software named HUGIN, and obtained the maximum causation chain of ship grounding accidents. Wang Mingda et al. (WANG et al., 2019) analyzed the text data of accidents from four aspects: human, machine, environment and management and identified the types of accidents by K-means clustering. The data model of multi-dimensional geophysical exploration operation accidents was established with the influence factors of accidents as the preceding item and the types of accidents as the latter item. The strong association rules between accident influencing factors and accident types were obtained by using multi-dimensional Apriori technology, and the occurrence rules of various accidents were discovered by analyzing these rules.

To sum up, at present, the scientists have carried out relevant research on accidents from the two dimensions of accident engineering application and accident basic theory. Although they have obtained certain theory and application value, these two research paths limit the development of upstream basic theory of accident science. it is also not conducive to promoting the development of safety science as a whole. Accident researchers often focus on their departments or industries and take specific accidents as the starting point to put forward new methods or solve new problems combined with accident analysis technology. There are several types of tendency characteristics: (1)the construction of accident cause model, (2)the new application of old accident model, (3)the transfer and application of other scientific theory, (4)the industry practice of self-scientific method. The above 
researches have not stripped the core essence of accident science, and have not formed the theoretical accumulation of accident itself. Accident science as a new cross-discipline, its discipline system remains to be improved, and its theoretical basis is still very weak. At present, the research on accidents, especially the exploration of mechanisms and laws, is more towards the perspective of risk, emergency and safety. A series of self-theories related to accidents, such as what is an accident, what is the nature of an accident, what is the characteristics, connotation and mechanism of an accident, and so on, are not unified and have a high degree of understanding. Studying accidents is a necessary topic for safety workers, and how to prevent or reduce accidents is also very important. Scholars who study accidents pay more attention to specific problems, rather than learning, do not pay attention to the promotion of accidents into a discipline. This is the malpractice of emphasizing transformation rather than research and application of theory at present.

The comprehensive and intersecting characteristics of safety science determine that it must be integrated with other disciplines in order to develop in the long term. The new accident theory should consider not only the achievements of management, sociology and political science, but also the theories of epistemology and philosophy to realize the security of different views of knowledge. In the context of the current complex social and technological systems and activities, it is no longer allowed to exist simple and linear security theories, methods and models to solve increasingly complex security problems. Modern social and technological systems are open "system of system", and their complexity and coupling are increasing day by day. For the security design and operation of the system, it has become an urgent scientific problem to study how to improve the security resilience of the system itself. It is difficult for traditional safety engineering methods to keep up with the development of new technologies and new technologies. These methods are based on a certain degree of predictability and decomposable assumptions. This is the disadvantage of the traditional method collectively known as "safety I". In view of the continued international attention to resilience engineering, finding a new breakthrough for safety science issues has also become a matter of urgency, resulting in a methodology called "safety II" that controls adaptation to unexpected situations. It is necessary to transfer the research of safety accidents from the surface research that promotes the analysis and prevention of system accidents to a deeper model and a more abstract dimension. The period of risk activity before the accident of the system is also the embodiment of the value of resilience.

At present, accidents are still deeply rooted in the hearts of the people all over the world. Accident researches are easier to be recognized by the society than the current safety-related disciplines. Thinking from the perspective of discipline construction, it is still possible to give new content to accident research. There are tens of thousands of domestic and foreign accident-related documents, which have long been in the shackles of the involuntary effect. So far, there has been a lack of improving accident research to the height of discipline construction. Accelerating the establishment of accident science has become an urgent requirement to build a basic theoretical system of safety science. This paper tries to discuss the basic problems of the establishment of accident science in order to lay a foundation for the development of the branch of safety science.

\section{The proposition of accident science}

From a global perspective, the current research frontiers and hotspots in safety science are relatively clear. Safety science is a cross and comprehensive discipline with the attributes of "synthesis of arts and science, intersection of disciplines and cross-cutting of industries". The study of safety science theory is to condense scientific issues and determine the dimension of combination. From the perspective of philosophical ontology, exploring the most essential and profound level of safety science is the original problem of safety. After an in-depth analysis of the core of safety, it can be found that the core of safety science is to reveal the law of occurrence and development of accidents and put forward accident prevention strategies. This indicates that safety science can also be called accident science, which is just the opposite description of the same problem. 
The research object of safety science is accidents, and the pursuit of safety is the pursuit of zero accident. The main goal of traditional safety work is accident prevention, so zero accident has become a ideal goal pursued by safety people and enterprises. As an idea or belief or pursuit, there is no doubt that zero accident can be advocated. If a certain range of time and space is preset and the nature of the accident is specifically defined, zero accident is completely achievable. However, if there is no definition of the scope of time and space, objectively speaking, zero accident is difficult to achieve, and it is also against the objective law to put forward the goal of zero accident without limiting the scope of time and space. Up to now, humankind' s knowledge of the objective world is still very limited, and there are still too many laws that are not clear in natural and life systems. Even if it is the man-made system and society created by human beings. human beings do not fully grasp the laws of their changes and the results of their emergence. However, due to the lack of subjective knowledge of human beings, as well as the high expectation, people are often overconfident and exaggerate their cognitive ability, which also leads to wrong cognition and decision-making. On this issue, it seems meaningful to treat accidents as a scientific discipline. Accident science enables us to obtain reliable evidence in practical applications in multiple safety fields, fill the gap between accident theory and applied research, and deepen attempts already discussed in traditional literature. The research on accidents requires multidisciplinary theories, and combine with different perspectives to achieve a complete presentation of the field. It is very important to have a comprehensive, accurate and profound understanding of the disciplinary nature and status of safety accident science. In order to determine the nature and orientation of safety accident science scientifically, it needs to be grasped from all - round and multi - Angle. Therefore, based on the relevant studies of safety science and accident theory, the basic issues such as the concept, connotation and research content of accidents are deeply analyzed and explained on the theoretical critical level, so as to provide new ideas for the basic research of safety science.

\subsection{Attribute relationship of accident}

Accidents can be given a variety of definitions in the literal sense. Understanding accidents needs to be traced back to the essential attributes of the accidents. In general, accident is defined as an unexpected incident that causes injury, which is based on the result of subjective thinking. Accident contradiction is one of the main contradictions that puzzle safety issues and guarantee production and life. How to clarify the nature of accidents and grasp the nature of accidents has become the primary subject of accident research. The solution of this contradiction depends on scientific and empirical knowledge. In the social system, accident research cannot simply rely on accident deduction, emergency management and cause analysis, and the acquired accident knowledge needs to conform to the essence of accident science. Overall, the accident attribute in accident science research is reflected in the following aspects.

\section{(1) Subjective abandonment}

Accident is a phenomenon that goes against people's will, which belongs to the object of exclusion. Here, will is the general term of people's general views or ideas of subjectively pursuing things such as safety, wellbings and health, and it is also understood as the goal of achieving safety. The subjective abandonment of accidents is its basic attribute, no matter which type of accidents, as long as people do not expect the situation will be abandoned by subjective individuals.

(2) Objective unexpectedness

Accidents are uncertain events, and the form of their occurrence is not only controlled by inevitability, but also affected by chance. When, where and why accidents occur are all unknown, and all accidents are unexpected. This embodies that the gestation, occurrence, development, transmission, coupling, migration and demise of accidents are uncontrolled natural processes. The accident can be divided into catastrophe accident and graded accident according to the speed when it occurs, no matter which kind of accident is the result of a comprehensive induction of a variety of accidental and inevitable factors. The process of the accident itself is the free development process divorced from human control, and the role of human in the early stage of the accident is 
reflected in the factors of unsafe behavior.

(3) System complexity

From the point of view of the system, the accident always destroys the point at a certain time and place in the system. The harmful event caused by any disturbance in the social system is the destruction of the internal sub-elements after the system's lack of resilience. The occurrence of accidents is systematic and the prevention of accidents is also a systematic project. In order to realize the system security and create the safety system, we need to take the overall philosophy as the basis, solve the essential factor problems as a breakthrough, and comprehensively consider the preventive measures after analyzing the causes of the accident.

(4) Governance iteration

Some of the causes of accidents are known and some are not. Some accidents can be prevented and some cannot be done. Some causes of accidents are controllable and some are not. It can be seen that the governance of some accident problems cannot be solved with the current technology or management. A "fly-fix-fly" approach is a new thinking paradigm that can be adopted. Paying attention to the prevention after the first accident is to eradicate the breeding bed of the next accident. Accidents in society are similar. After studying a large number of causes, types and measures of similar accidents, it can be found that there will be problems that can not be solved by existing technical means or management intervention in the process of managing similar accidents. Only thinking in front of the accident can block the road caused by the accident. Therefore, the iterative and updated thinking should be used to solve the accidents that may occur in the future.

(5) Causal factor complexity

In the early days, human beings classified the causes of accidents as natural punishment and natural calamity, and a series of theoretical models appeared in the middle stage. Up to now, the development of accident cause theory has been progressively improved. From frequent tendency theory to Heinrich model and from energy release theory to system cybernetics, the development of accident causes is driven by the complicated causes of accidents. Different accidents may be caused by the same cause, and different causes may also explain the same accident. This complicated relationship is caused by the increasingly complex social technology system, rapid industrialization and human speculation. New technologies, new processes and new systems will inevitably bring about new factors that cause accidents, and the factors that cause accidents are also full of changes.

(6) Incident process variability

The changes after accidents in simple systems are predictable, observable and controllable. The more complex systems are affected by internal and external factors, the higher complex the accident process. Accidents are prone to coupling, migration, diffusion, and transmission after they occur. These phenomena have become one of the important hotspots in accident studies. Many new phenomena in accidents have also aggravated the physical and psychological trauma of humans today. A series of social problems afterwards came into being. All these are brought about by the variability of the accident process, therefore, speeding up the study of the accident process will become the focus of future research.

(7) Scientific foundation

All safety science originates from accident research. Human beings begin to explore the safety world by paying attention to accidents, and the study of safety science is completed under the fundamental purpose of accident prevention. But safety science is often mistakenly extended to industry safety and become a panacea. Accidents are the original, safety is the form. Safety science is a methodology to prevent accidents, and it is to prevent all kinds of accidents. Accident science is a science with a larger category, deeper foundation and stronger theoretical than safety science. Taking accidents as the research object can form a truly independent discipline by using scientific methods, which makes safety science more specific and practical.

(8) Potential concealment

Everything in the world is in a variety of risks and hidden dangers all the time, especially in the field of 
human activities. The danger does not necessarily lead to an accident, but it must form the potential conditions for the occurrence of the accident. According to this understanding, accidents can be divided into hidden accidents and explicit accidents. The former is the sum of the various dangerous elements that are not expected to appear, and the latter is the actual accident in which the injury has occurred. General potential hidden elements may lead to accidents, but whether the accident occurs is affected by inducing factors, and the potential concealment of accidents will be the basic attribute of accident prevention and advanced management.

Accident science can be regarded as a comprehensive science under the intersection of multi-sciences, and accident science requires the interdisciplinary integration of sociology, management and engineering. Various management models can provide theoretical reference and guidance for accident cause models. sociological methods provide a supporting foundation for accident prevention. As shown in Figure 1, it is a brief illustration of the cross-attribute of accident science.

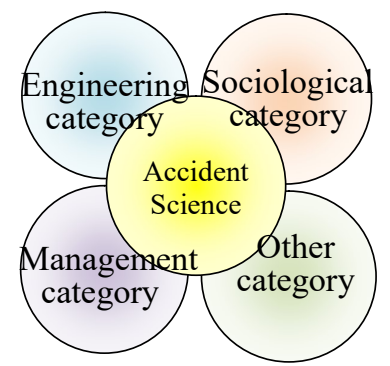

\subsection{Characteristics and connotation of accident}

Fig.1 Comprehensive interdisciplinary attributes of accident science

(1) There must be its own characteristics or laws in accident research. Only by understanding the characteristics of accidents can effective measures be taken to prevent accidents. According to the above accident attribute relationship, the basic characteristics of accidents can be summarized as follows: (1) Occasional loss. The severity of the accident consequences is random and unpredictable. Repeated similar accidents do not necessarily have exactly the same consequences. This explains that the consequences of similar accidents are completely different, which puts forward the idea of symptomatic treatment for the prevention of accidents. (2) Systematic process. Whether it is a general accident or a special accident, it is the result of the disruption of the internal elements of the system. The causes of accidents are various, and the measures to prevent accidents are also comprehensive. the management of accidents should be treated in accordance with the idea of system engineering. (3) Complexity of prevention. The occurrence of accidents is characterized by suddenness and accidents. Although some accidents have a gradual change process, it is difficult for people to decide where and when the critical point of accidents occurs. There are many uncertain factors for accident prevention, which are determined by the complexity of the accident prediction process. (4) Transmissibility of change. After the occurrence of an accident, the system does not simply end with the consequences of loss, but forms a chain or coupling phenomenon through a series of changes and transmission. The occurrence of the accident can be regarded as the propagation process of a series of interactive elements, and there is also a ripple effect after the accident. For other processes of the system, it is necessary to establish a closed-loop system, especially the system or part where the accident is located, to maintain functional connection and structural independence with other systems. (5) Objective causality. All accidents have their causes, and the causes of most accidents can be recognized by people. It is not easy to find the causes of an accident in a more complicated accident. Many accidents are affected by multiple factors. So it is necessary to deeply analyze the root cause of the accident, find out the root cause of the accident, and study the relationship between cause and effect to prevent the accident from happening again. (6) Potential prediction. The so-called potential prediction of the accident means that the hidden danger of the accident exists in the gestation stage at the beginning of the development, and the way of existence is generally hidden and latent. With the random change of each process of production, the accident gradually develops to dominance. Hidden dangers in a latent accident have existed for a long time, waiting for a certain time or condition to happen. Premonitory manifestation occurs during the incubation process, which is rarely perceived or recognized. 
(2) The connotation of accident is the abstract nature concept of accident. according to the social system, accidents can be divided into macro accidents, meso accidents and micro accidents. Macro accidents are system accidents formed by the combination of several medium systems composed of the most complex small systems, such as coal mining industry accidents or oil mining industry accidents and so on. The meso accidents are accidents caused by a system with many elements and complicated internal connections and difficult centralized control. And meso accidents are composed of many small system accidents. such as an internal operation accident of an enterprise, the collapse of a large-scale construction project or the explosion of a city pipe corridor, and so on. The micro accidents are caused by systems with few elements and simple internal connections, such as the failure of a machine, the injury of a team member and so on. The connotation of the macro accident is more complex, which involves the analysis of the elements of the accident system, and the accident occurs as a result of the comprehensive action of the man-machine loop management within the system. The basic connotation of social system accidents includes the followings. (1) People are always at the core of social accidents. The internal control and intervention of the system are the result of human action, and the realization of the system function and ensuring the safety of the system are decisions made by human participations. In the accident, human cognition and behavior will affect the development and trend of the accident, and the spread and coupling of accidents in the system are also affected by people. (2) The accident itself has certain system characteristics. An accident is a state in which the various elements of injury are coordinated under a certain morphological structure. The theoretical aspects include the characteristics, structure, evolution and methods of the accident system, and in practice, under the premise of accident epistemology, methods, techniques and laws that can be used in the field of safety and can solve accident problems. (3) The accident is the inevitable result of the increase of system entropy. Everything is between the equilibrium state of disorder and order, but entropy is the inherent attribute that leads to the destruction of system balance, and the increase of entropy means that the safety state of the system is challenged. If this non-equilibrium state is not corrected, a new entropy flow will be formed and accidents will occur. Thus, measures should be taken to prevent the negative effects of entropy increase, so as to prevent accidents. The characteristics and connotation of the accident are briefly descripted in Table 1. 
Table 1 The characteristics and connotation of accident

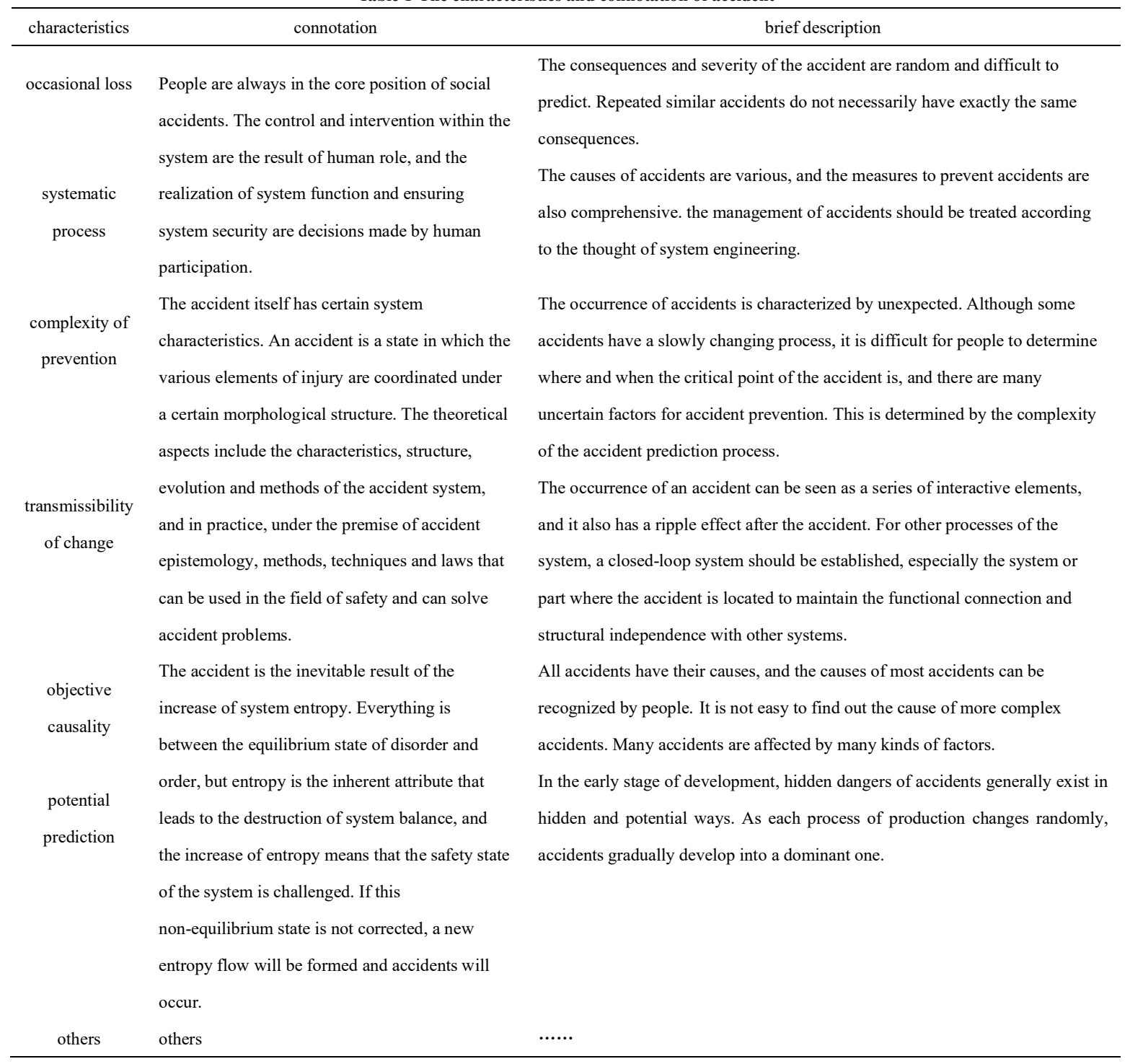

\subsection{Types of accidents}

In order to understand and study the accident, it is necessary to classify the accident, which is based on different purposes, methods and angles. In reality, there are more than a dozen classification methods including accident attributes, hazardous consequences, industry, injury, severity, national standard, occurrence cause, injury nature, operation nature and so on. No matter which kind of accident classification needs to be based on the needs of research and practice, combined with the appropriate selection of classification methods in line with the actual situation in accident management. The scientific classification of accidents can find out the regularity of accidents, and then put forward the general prevention methods of similar kind of accidents. This article proposes a systematic method of accident classification from the perspective of research. Based on the cognitive process of exploring things, the accident is classified into five layers including knowledge layer, technical layer, theoretical layer, management layer and logic layer, and there are corresponding subcategories under each category. Among them, the knowledge layer is the content category of accident research, the technical layer is the process of accident research, the theoretical layer is the basic theory system of accident research, the management layer is the method of accident management, and the logical layer is the basis for determining the accident object. The specific hierarchical relationship of accident cognitive classification 
is shown in figure 2 .

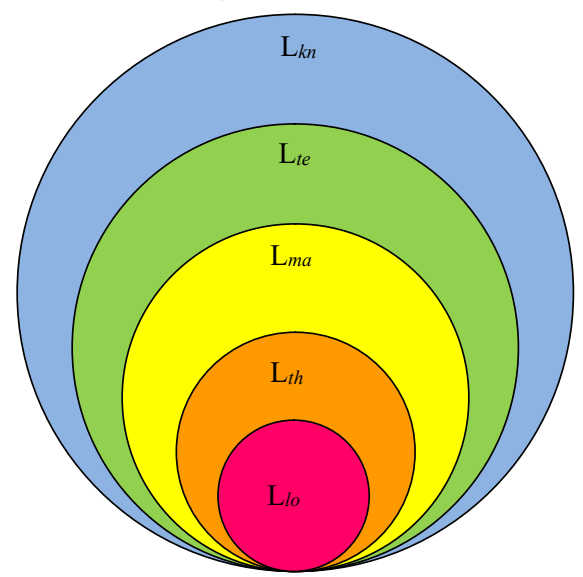

$\mathrm{L}_{l}{ }^{\bullet}$ Logical layer: Philosophical cognitive logic, accident concept development, causative thinking construction, accident prevention paradigm

$\mathrm{L}_{t h}{ }^{\bullet}$ Theoretical layer: Accident model architecture, probability quantization equation, evaluation grading method, prediction and warning procedure

Lma Management layer : System control strategy, human behavior management, material energy regulation, environmental and external intervention

$\mathrm{L}_{t e}{ }^{\bullet}$ Technical layer: Monitoring and testing equipment, accident early warning system, emergency rescue system, personal protective equipment

$\mathrm{L}_{k n}{ }^{\bullet}$ Knowledge layer: basic subject theory, accident system engineering, management technology optimization, experience and practice summary

Note: The accident itself can belong to the philosophical level or the scientific level. From the perspective of studying the nature of accidents, it is generally believed that the classification of accidents in different dimensions is a study with certain scientific value. This level of relationship is also to discuss the classification relationship of accidents in the cognitive process, and clarifying the relationship of accidents can more deeply study the nature of accidents.

\subsection{Accident association}

Fig.2 The hierarchical relationship of cognitive classification of accidents

Accident science covers a wide range of areas, and the breadth and depth involved are extremely profound. The contents of accident science are the fundamental elements to promote the development of safety science at all levels of upstream, middle and downstream. Based on the analysis of accident science, there are five levels of logical associations: accident systematic system, accident person subsystem, accident material subsystem, accident environment subsystem and accident management subsystem. And accident science has three levels of realistic relevance: prevention in advance, handling during the event and recovery after the event. Fig. 3 (a) and (b) show the related characteristics of accident science research, which shows that accident science is an unavoidable problem both in logical thinking and in practice.

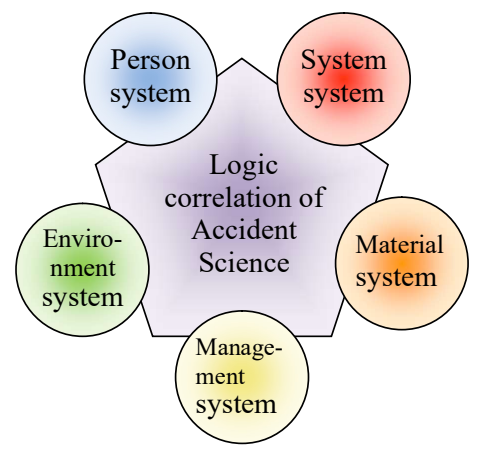

(a) The Logical Associations of accident science

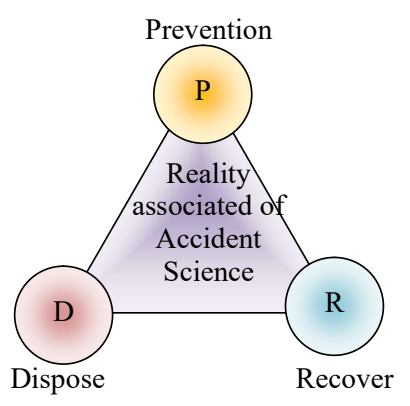

(b) The Realistic Associations of accident science

Fig.3 Associated characteristics of research on accident science (a)\&(b)

\section{Related concepts of accident science}

\subsection{New definition of safety}

Safety means no accident, but no accident does not mean safety. Accidents are the origin of safety research, and safety is an abstraction based on accidents. In order to study the problem of accidents, we must first clarify the basic concepts of the relevant disciplines, and in the category of safety science, it is urgent to analyze the new definition of accident science based on the thinking paradigm of accident. According to this thinking, some new vocabulary understandings of new disciplines are established, and the concept of subject in the field of safety science can be 
restated with accident thinking according to the science guidance. Referring to the literature at home and abroad, we can see that the concept of safety discipline is not unified so far, and scholars understand the concept of safety from different angles. Basic concepts guide the establishment of basic theories. At the discipline level, the primary task is to clarify the basic concepts, and the expression of the main safety concepts and connotation interpretation are shown in the followings.

Liu Qian, one of the predecessors in the safety field in China, put forward a scientific definition of safety. "Safety is the existing state (or health status) in which people's body and mind are protected from external factors and its guarantee conditions" (LIU, 2010). The definition strictly summarizes the nature and characteristics of safety. Based on this, a new definition of safety is proposed. Safety is an activity carried out in order to enable rational people to achieve a certain intention within a certain scope of time and space, and through the adoption of management and technical means, it achieve the comprehensive state and guarantee conditions of rational people's physical and mental well-being, stability of system elements, control of organizational functions, prevention and resolution of risks. The basic connotation of the new definition is as follows.

(1) The new definition is a logical refinement under the discipline theory of accident science, reflecting the characteristics of accidents. The foregoing shows that the accident has the characteristics of system process and occasional loss, and taking accident science as a starting point to explain that the basic definition of safety reflects more emphasis on the core of safety research, highlighting that the fundamental problem of safety research should be accident. It is an attempt to refine the safety problem in conjunction with accident science, which can make a further discussion on the accident-related theory. From the new definition of safety, it can be seen that safety is still a comprehensive state and a set of conditions to guarantee this state, which is not a simple application but is clearer and more specific than the traditional definition. Showing the state problem is not only about the physical and mental harm of human beings, but also extends to the areas of factor stability, organizational control, risk mitigation and so on. The reason for expanding the scope of the new concept is to take into account the systematic process and prevention complexity of the accident.

(2) Define the conceptual subject category and clarify the research boundary. The rational person mentioned in the definition is different from the irrational person, and the general accidents are often caused by the behavior of the rational person. The rational person mentioned here is a person whose cognition, experience, perception, consciousness, attitude, emotion and personality characteristics under normal state meet the statistical state of most people. Such as mental abnormality, chaotic thinking, visual and auditory hallucinations, passionate crimes and minors without civil capacity can be categorized as irrational people. Rational people are very important in social life, especially for ensuring system safety. The definition of safety defines the subject nature of human beings, which also shows that security research must be considered within a certain range of elements. Otherwise, the lack of a clear boundary will easily lead to chaotic scientific definitions.

(3) Determine a certain category of time and space to provide assumptions for the content of safety research. Ontologically speaking, if there is no limitation of time, space and nature, accidents exist objectively and disappear without human will. So far, human understanding of safety science is still very limited, and human beings have not fully grasped the changing laws and various emergence results of accidents. According to the epistemological and ontological viewpoints and the theory of complex systems science, accidents actually occur in the form of emergence, if a certain field of time and space is not defined, that is, the scope of discussion is not defined, then safety problems will always arise, which is an inevitable objective law. Safety has explicit value only in a certain field of time and space, and the problems of accident science can also be recognized and understood.

(4) Human activity is one of the basic connotation of safety, and there is no accident without activities to achieve a certain purpose. No matter what kind of system it is, as long as people participate, some activities will take place, and the elements of the system are always dominated by certain activities. In a broad sense, human activities are a series of activities of different scales and types in order to survive and improve living standards, including agriculture, forestry, fishing, animal husbandry, mining, industry, commerce, transportation, tourism and various engineering construction. 
Human is a constituent element of the system. In recent hundreds of years, the irrational overspeed development of human society has made human activities become the dominant negative factor affecting the security of various systems in the society. Such as accidents in high-risk industries, accidents in social life, damage to system and failure of personal safety protection, all of these have sounded the alarm for human safety. Therefore, some human activities must be taken as the prerequisite when studying the safety core of accident science.

(5) Management and technology are the core of safety methodology. From a broad perspective, all accident prevention methods are nothing more than engineering and management. The safety problem is a comprehensive system problem involving many aspects in the whole field, and comprehensive measures must be taken to solve the safety problem. Accidents are caused by complex factors or chain reactions. therefore, comprehensive and systematic measures must be taken for accident prevention. Only by adopting a two-pronged approach to cure both the symptoms and the root causes can we achieve immediate results. Accidents will inevitably involve human participation, and the study of human beings is inseparable from the means of management. the theories of sociology, management, politics, psychology and behavior play a core role in individual control. The operation of the material (device) in the system is inseparable from the blessing of the existing technical means, in which electronic and electrical technology, computer technology, mechanical material technology and "four new" play a fundamental role in control of material. Therefore, the study of accident science and the definition of new safety from the most important human and material factors in the system requires a dual approach of management and technology.

(6) The core content of the new safety includes four aspects: physical and mental well-being, stability of system elements, control of organizational functions and prevention and resolution of risks. The safety of human body and mind is the basic pursuit of safety. The traditional definition of safety is to take the body and mind as the research object, which involves the body, that is, the physical body is healthy and the external psychological is noninvasive. Physical health means that the body is not subject to sudden injuries (occupational injuries and life accidents) from outside, and chronic occupational diseases are also included. Psychological non-invasive refers to the long-term and intractable secondary cognitive impairment caused by internal accidents (such as psychological shadow, nightmares, false hallucinations). The new safety definition refers to this part of the research as transforming accident people. The stability of system elements refers to the stability mechanism and method against accidents from the point of view of system resilience. The content includes four aspects: system structure stability, system function stability, system risk stability and system element stability. One of the connotations of new security is to take system engineering as the paradigm to promote the maintenance of system stability. The control of organizational function means that the basic functions of social organizations are controlled to a certain extent in the process of realization to prevent functional disorders and morphological spillover. Preventing risks is one of the new missions of new safety, and defusing risks is also the content of safety research. New technologies and management measures are introduced in the study of how to determine risk factors, risk classification and risk control.

(7) The new safety emphasizes the comprehensive state and its guarantee conditions. Safety is a comprehensive concept, which can not only reflect the subjective feelings but also portray the objective state. Whether the condition is good or not is determined by the internal and external system conditions. If the external conditions can ensure that the people, elements, organizations, and risks of the system are under control, then it is called a safety system. The ultimate goal of the new safety is to achieve a normal comprehensive state of the system or to have various conditions under which the state of the system can be guaranteed. This provides many options for expanding the field of accident science. The realization of the state or condition must rely on the advancement of the discipline, so speeding up the construction of the discipline is very important to reveal the safety laws.

(8) The proposition of the concept often needs to dig deeply into the essence behind the concept and put forward the conclusion which conforms to the law of science. The new safety definition proposed by this paper has high value from the perspective of academic research. With the deepening of research, the connotation of its definition is constantly enriched and improved. Therefore, it is necessary to have a certain scientific vision and acuity, and 
researchers should grasp a series of safety science problems derived from accident science to understand safety science from a higher level.

\subsection{Basic concepts}

According to the new safety definition and its connotation, a series of basic definitions of safety science can be concluded. Table 2 shows the new definition and connotation of important basic terms in the field of safety science, from which we can see that a series of concepts based on accident thinking are necessary and valuable for the construction of basic accident science. Only after clarifying the concept can we provide ideas for the basic research of accident discipline and avoid impracticality.

Table 2 Basic concepts of Accident Science and their expressions based on the new safety definition

\begin{tabular}{|c|c|c|}
\hline concept & definition & connotation \\
\hline safety & $\begin{array}{l}\text { Safety is the comprehensive state and } \\
\text { guarantee conditions for rational people to } \\
\text { adopt management and technical means in the } \\
\text { process of realizing a certain intention in a } \\
\text { certain time and space to achieve the physical } \\
\text { and mental well-being of rational people, the } \\
\text { stability of system elements, the control of } \\
\text { organizational functions and the prevention } \\
\text { and resolution of risks. }\end{array}$ & $\begin{array}{l}\text { The new safety definition has eight important } \\
\text { connotations. See above. }\end{array}$ \\
\hline risk & $\begin{array}{l}\text { Risk is the possibility and severity of harm to } \\
\text { rational people in the system in a certain time } \\
\text { and space. }\end{array}$ & $\begin{array}{l}\text { The possibility and severity of damage to the } \\
\text { elements in the system not only determines its } \\
\text { state, but also reflects the security state of the } \\
\text { system. It can be described qualitatively and } \\
\text { quantitatively. }\end{array}$ \\
\hline accident & $\begin{array}{l}\text { Accident refers to the harmful results that lead } \\
\text { to the physical and mental damage, the } \\
\text { destruction of system elements and the failure } \\
\text { of organizational function of rational people in } \\
\text { a certain time and space. }\end{array}$ & $\begin{array}{l}\text { Accident can be understood as a state or event, } \\
\text { which generally produces undesired } \\
\text { consequences and has a systematic destructive } \\
\text { effect. The accident will inevitably lead to } \\
\text { certain negative consequences. }\end{array}$ \\
\hline danger & $\begin{array}{l}\text { Danger is the state in which the system may } \\
\text { be endangered in a certain time and space and } \\
\text { the lack of safeguard conditions or a measure } \\
\text { that may lead to negative consequences. }\end{array}$ & $\begin{array}{l}\text { Danger can be quantitatively described by the } \\
\text { degree of danger. Danger can be regarded as the } \\
\text { actual premise of risk. In the system, the danger } \\
\text { may be caused by the unsafe behavior or unsafe } \\
\text { state of the system elements. }\end{array}$ \\
\hline $\begin{array}{l}\text { hidden } \\
\text { danger }\end{array}$ & $\begin{array}{l}\text { The hidden danger is the unsafe behavior of } \\
\text { all people who may cause danger in a certain } \\
\text { time and space, the unsafe state of things, or } \\
\text { the defect of management. }\end{array}$ & $\begin{array}{l}\text { Including hidden dangers of the system, hidden } \\
\text { dangers of the system elements and external } \\
\text { hazards, unsafe behaviors of people, unsafe } \\
\text { states of things, bad environment and } \\
\text { management defects all belong to hidden } \\
\text { dangers of the system elements. }\end{array}$ \\
\hline $\begin{array}{c}\text { Source } \\
\text { of danger }\end{array}$ & $\begin{array}{l}\text { The hazard source is the sum of the internal } \\
\text { and external factors that can form the } \\
\text { systematic risk hazard in a certain time and } \\
\text { space. }\end{array}$ & $\begin{array}{l}\text { The actual source of danger may exist, but it } \\
\text { does not necessarily bring danger, or even an } \\
\text { accident. Hidden danger must be a source of } \\
\text { danger, and the source of danger is not } \\
\text { necessarily a hidden danger. }\end{array}$ \\
\hline
\end{tabular}

\section{Basic problems of accident Science}

\subsection{The definition of accident science}

Safety is an ancient topic, but accident science is emerging as one of the safety disciplines. In the national standards for discipline classification, many safety disciplines are in urgent need of improvement and development. Accident science is a virgin land for basic research. The purpose of the establishment of accident science is mainly to clarify the confusion of the basic theory of accidents and form a consensus on accidents. This paper discusses the subject boundary and research category of accidents and establishes the conceptual model of safety accident science from multiple perspectives. According to the aforementioned accident concept and the purpose of accident science, and 
based on the interpretation of several concepts in safety science, the definition of accident science can be summarized. Under a certain category of time and space, accident science is a comprehensive cross-discipline, which taking safety as the purpose and the accident as the research object studies the mechanism and law of accident cause, evolution, disaster or disappearance, accident prediction, prevention optimal control methods engineering measures in the management and technical means of emergency, exploration, identification, treatment and reference of accidents. It is simply understood that accident science is a safety scientific theory that studies the law, management and technology of accidents in the system. The conceptual model of accident science is shown in figure 4. Through the construction of the conceptual model, the research purpose, research object, research content, theoretical basis and discipline objectives of accident science can be clearly defined, which lays a foundation for exploring the development of accident science.

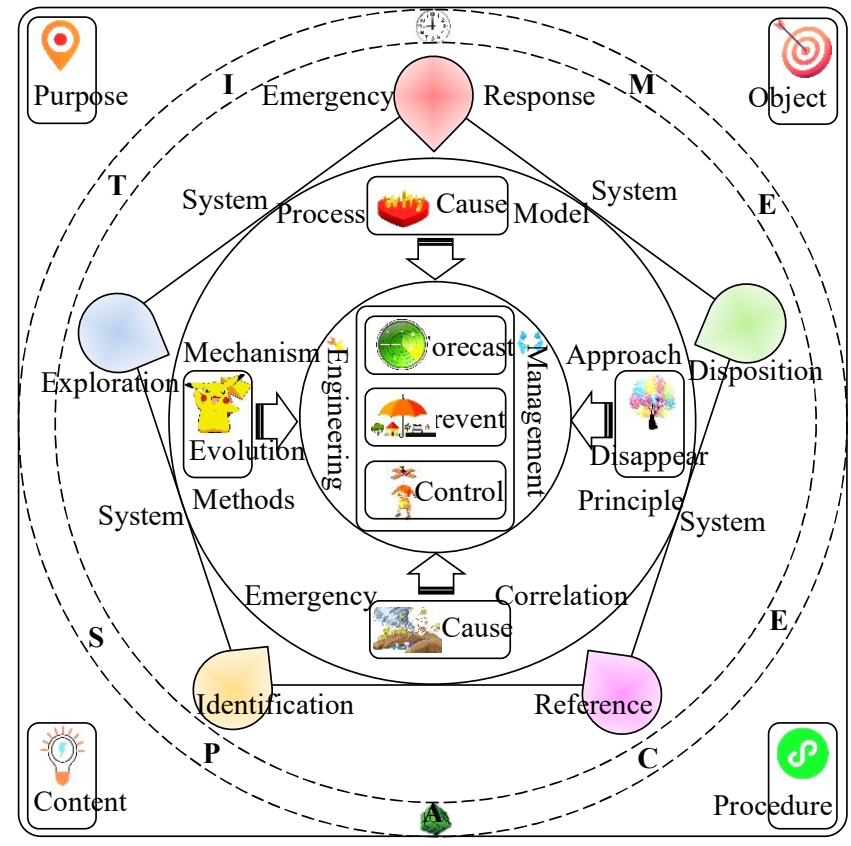

Fig. 4 Definition model of accident science

\subsection{Connotative explanation of accident science}

Accident science is a comprehensive and interdisciplinary subject. Solving subject problems requires the support of multidisciplinary theory and the comprehensive application of scientific principles to guide the establishment of accident theory. According to the accident definition model above, it can be seen that the concept of accident has important connotation and denotation. The concepts and subject connotations involved in accident science are explained as follows.

(1) The definition of accident discipline describes the basic framework of accident science. From the conceptual model above, we can see the purpose, object, content, procedure, method, theory and disciplinary framework of accident research. In the concept, a certain scope of time and space is defined for the study of accident science, which provides the basic conditions for the subject construction to limit the assumption and materialize the research object. System as one of the objects of study reflects that accident science exists in order to solve system problems, which is the mission entrusted by history. After the long-term development of accident theory, it has gradually formed a scientific hypothesis in line with human scientific cognition and reality. The trend of systematic research also promotes the transformation and upgrading of safety science. The research purpose of accident science is to achieve system safety, and this purpose is also the endogenous driving force for the construction of new disciplines. It is important to understand that the purpose and object of research is the philosophical scientific problem that must be solved in the establishment of accident science.

(2) What is the " $3-4-5$ " model of research content? It can be seen from the model diagram that the research content of accident science is very complex and in-depth, and the structural research content can be roughly divided into 
three layers. That is, the outer layer includes emergency, exploration, identification, treatment, reference and other programs and technical means. The middle layer includes engineering or management measures for the cause, evolution, disaster or disappearance of system accidents. The inner layer includes the prediction, prevention and control methods of an accident. The research content of accident science is the core of the concept of accident science, which supports the development and improvement of accident theory. The following points are the specific explanation of the research content.

(3) The " 3 " of accident science refers to the prediction, prevention and control methods of accidents of the inner layer, which is one of the core contents of accident research. Accident prediction is a comprehensive measure that uses prediction methods to analyze the risk and risk degree of accidents, then to provide managers with accident risk, accident severity assessment and early warning of accident consequences. Accident prevention is an accident management method based on prediction. Combined with the results of prediction, a series of engineering and management programs are put forward to prevent the occurrence and development of accidents. Accident control is to learn from the management control theory to strengthen the control of various elements in the accident management system, in order to achieve the purpose of accident prevention, emergency and safety. The important part of accident research is prevention in advance, emergency in the event and prediction after the event. Generally speaking, it is the theory of control methods for accidents.

(4) The "4" model of the research content is the engineering or management analysis of the cause, evolution, disaster or disappearance of accidents in the system. Through the cause analysis, the cause, rheology, derivation and coupling relationship of the accident are obtained. Accident evolution has also been a research hotspot in recent years in accident studies, because the more complex the system is, the easier it is to produce the evolution and change of accident. This evolutionary process is an important cause of derivative accidents and chain accidents. The cause analysis of disaster is caused by the disaster effect after the accident. The small accident evolves into a big accident, and the big accident evolves into a disaster. Both natural disasters and man-made disasters can be caused by the disaster-causing effect of complex accidents. Accidents also have the stage of disappearance, no matter which link of the life chain of disasters and accidents can take measures to cut off the development of accidents, and finally make the accidents disappear. These are the specific description of the "4" model of accident studies in the middle layer, which is also a bridge connecting the outer layer and the inner layer.

(5) Specifically, the "5" of the content model is five aspects of emergency, investigation, identification, treatment and reference. Among them, emergency is the process of rescue and treatment after the accident, which is not only the product of traditional post-event management thinking, but also a common practical method of accident management. Investigation is a specific preliminary work carried out after the accident, which provides a preliminary reference for search and rescue at the scene of the accident. Identification is the basic process of analyzing the cause of the accident by using the materials such as personal evidence and material evidence at the scene of the accident. Treatment is to put forward technical and management measures for the parties and matters of the accident according to the analysis of the nature and cause of the accident. Reference draws lessons from the knowledge and experience of similar accidents in accident analysis and prevention, and provide cases for the specific disposal of accidents. The outer layer model of accident science is the focus of modern safety science research. With the development of history to modern society, accident research is moving towards the direction of law and mechanism, and future accidents are bound to develop towards the core of prediction, prevention and control. The above three points are based on the accident science research content of the " $3-4-5$ " model, which aims to promote the further and scientific development of accident science research.

(6) A comprehensive cross-discipline based on engineering measures and management measures. Accident science is the underlying basic research field of safety science, which is guided by the methods and principles of safety science under the framework of safety science. Safety science is a large cross and comprehensive science, and the content involved is very complex. It roughly includes the theoretical support of more than ten basic disciplines, such as 
science, engineering, management, law, liberal arts, medicine and so on. The attribute of cross-discipline determines that the study of accident science must find a new way in accordance with the law of accident science on the basis of reference and integration. The basic theory of accident science is not a self-system with its own profound scientific accumulation, but another system that needs integration and innovation. The methods, principles, procedures and systems of accident science research are constantly absorbed and innovated in the development of the discipline. The study of accident science is inseparable from human beings, who are not only the parties to the accident but also the decision-makers of the accident. The research involving people is not a simple problem in the field of technical engineering, but a comprehensive complex body that must carry out psychological, physiological, behavioral and cognitive research. such research requires the multi-disciplinary intervention of engineering measures and management measures.

(7) Generally speaking, the concept of accident science is a preliminary exploration, which is of fundamental significance for follow-up in-depth research. There is almost no special precedent for systematic research on accident science at home and abroad. after thinking deeply about the current development situation and future trend of safety science, the author decided to conduct a systematic study of accident science. Truth is always developing, and there is no absolute exhaustion of truth. The basic concept of accident science is also inadequate or even wrong, which needs to be constantly improved and condensed thinking. The development of safety science is a spiral process in China, and countless scholars should be admired for their corresponding contributions from their respective fields. The author hopes that the conceptual model of accident science established above can also be regarded as a small innovation of safety science and provide a new method for follow-up research.

\section{The theoretical basis and discipline classification of accident science}

\subsection{The theoretical basis of accident science}

As one of the basic disciplines of safety science, accident science also has the basic attributes of safety science, and accident science also has the characteristics of comprehensive and interdisciplinary science. Throughout the development of other disciplines, we can see that many classical basic disciplines (majors) are produced through a certain form of synthesis on the basis of intersection. The interdisciplinary intersection of accident science is a comprehensive intersection involving multi-level relations, including the basic interdisciplinary of the outer layer and the related disciplines of safety science in the middle. Accident science is mainly related to safety behavior, safety evaluation, safety psychology, safety management, safety ergonomics, safety law, safety systems, safety pedagogy, safety monitoring and monitoring, safety planning, emergency management engineering and industry safety engineering. Through the intersection of the above disciplines, accident science has a theoretical basis based on cross-disciplines, and forms its own theoretical system under the theoretical basis of other disciplines. The main theoretical basis of accident science is law, psychology, pedagogy, behavioral science, management, economics, systems, mathematics, physics, chemistry, biology, logic, investigation, technology, engineering and so on. These basic theoretical disciplines are combined with safety to form a safety-based discipline, and then become the basis of accident science.

The theoretical basis of accident science is complex and diverse. Based on different research perspectives, targeted basic theories can be obtained, which are combined with different dimensions of accident research to form a model under the background of accident discipline. For example, the cheese model in management has become one of the accident cause theories when it is transferred to accident research. Monte Carlo simulation algorithm in system science can be used to calculate the occurrence probability and accident risk of system links. The application of behavioral cognitive theory in accident research is the cognitive processing process of safety information, and the resulting information distortion leads to the model of accident. The psychological effect of psychology on the decision-making mechanism of people's unsafe behavior in accidents is also the key content of accident science research. Evaluation methods in technical science are widely used for risk assessment and hazard grade determination, and so on. Therefore, accident science needs the theory of various disciplines as a support. In fact, the establishment of the discipline of 
accident science is the innovative exploration of the discipline. Specifically, the interdisciplinary relationship of accident science is shown in figure 5 .

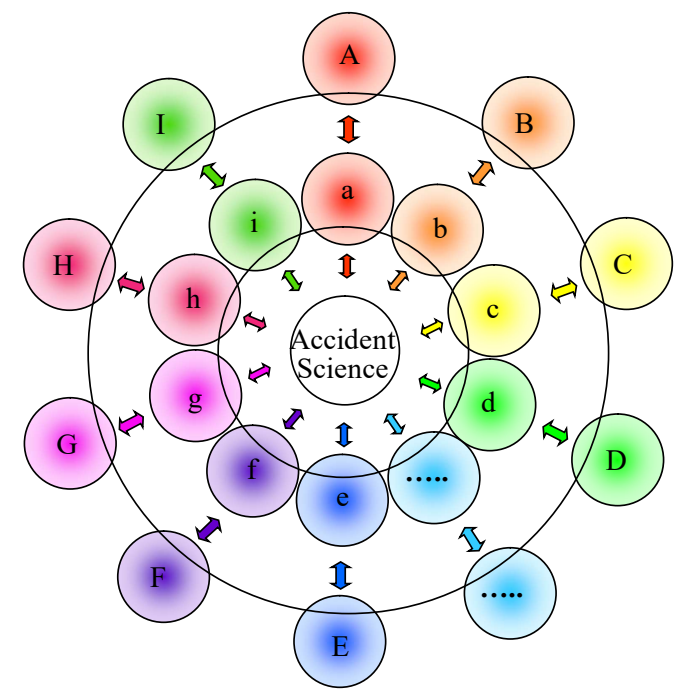

Note:

A: behavioral science; B: management; C: ergonomics; D: law; E: system science; F: economics; G: pedagogy; $\mathrm{H}$ : psychology; I: technology, a: safety behavior; b. safety management; c: safety ergonomics; d: safety law; e: safety systematics; f: safety economics; g: safety pedagogy; h: safety psychology; i: safety assessment

\subsection{Discipline classification of accident science}

Fig. 5 The theoretical basis of accident science

At present, there are few disciplines related to special accidents in the Classification and code of disciplines, and there are many blank disciplines in safety science that need to be supplemented and improved. Accident science as a new discipline still lacks the necessary theoretical system, so its research content is comprehensive and complex. First of all, the establishment of the discipline system needs to be guided by the foundational disciplines, to study the further classification and refinement of the discipline branches, and to learn from the natural science system to complete the enrichment and perfection of the discipline branches of accident science. According to the research field and definition of accident science, it can be seen that it has an independent discipline system. Accident science is formed by the cross integration of safety science system, natural science system and management science system, and subordinates to the foundation of safety science. The discipline of safety science has a wide range of research fields and branches, and the accident science as a branch discipline of safety science have a rich discipline system. On the basis of learning from the attributes, levels and research objects of different disciplines of safety science (WU, 2007), this paper attempts to enumerate the discipline branches of accident science combined with the research objects of accident science. The branches should at least include: accident psychology, accident cause theory, accident evolution law, accident prediction and prevention methods, accident emergency management, accident investigation methods and techniques, accident simulation and reproduction technology, accident identification technology and norms, accident control engineering, typical accident cases, accidents in a certain industry, etc. The established tree of accident science is shown in figure 6. 


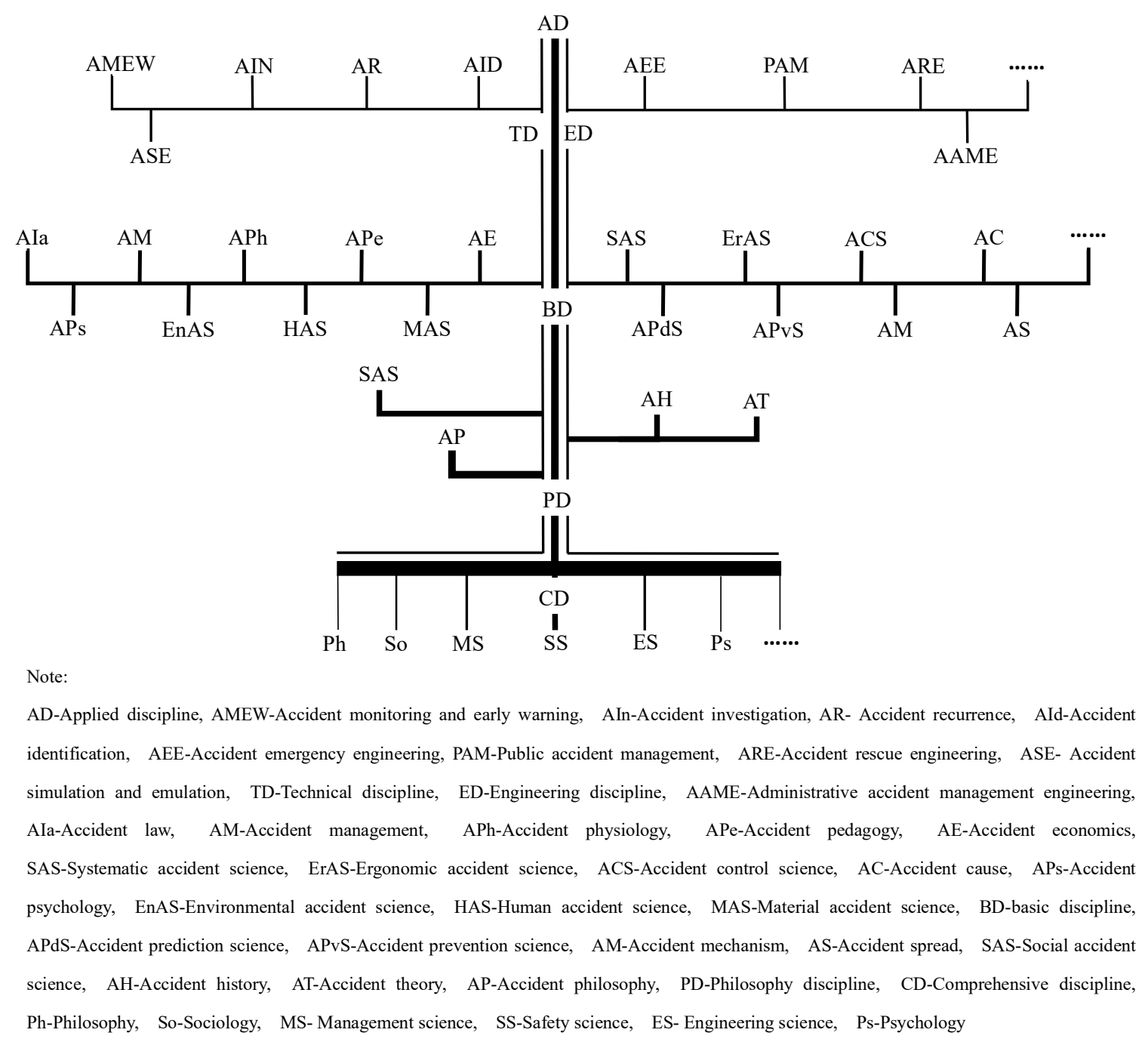

Fig. 6 The tree of accident science

\section{Research content of accident science}

\subsection{The research content of accident science}

From the definition of accident science, it can be concluded that the research content of accident science is the "3-4-5" model, which is divided into the aspects of accident prediction, prevention, control, engineering or management analysis of accident cause, evolution, disaster or disappearance, emergency response, exploration, identification, treatment and reference. To sum up, the research contents of accident science are mainly classified into the following three aspects.

(1) Research on accident theory

In the study of accident science, the most basic and important task is to analyze the cause of the accident and the mechanism behind the accident. The accident is the result of the comprehensive influences of many system factors. With the increasing complexity of the social and production system, the variability and complexity of hidden dangers are also increasing. The control and management methods of sources of danger also urge managers to think about how to achieve safety management, on the basis of which a new understanding of accidents is formed. The cause model has existed for a long time, but the accident theory which accords with the characteristics of the period is seldom. One of the research contents of accident science is to excavate the accident cause theory and accident mechanism. 
(2) Research on accident application management

As an unexpected result, accidents rarely involve the technical knowledge of accidents, but as a discipline, a theoretical system and methodology in line with the applied technology of accidents should be established. The ultimate goal is to use the accident principle and model to explain the process of the accident and to use the accident analysis method to study the law of accident development. In the aspect of analogue simulation, further research is also needed to provide scientific guidance for emergency rescue and prevention. The prevention organization strategy before the accident, the emergency management method in the accident and the improvement and promotion plan after the accident are all the practice of accident application management.

(3) Basic researches on the discipline of accident science

The basic contents of accident science, research framework, research objectives, accident science principles, accident science methodology, accident science procedures, accident strategy analysis etc. are involved. The study of the discipline is more from the perspective of scientific thinking to use a systematic method to construct the disciplinary basis of accident science. Based on principles, methodology, conceptual framework and practical methods, the theoretical research of accident science draws lessons from the framework and construction models of other safety science disciplines, then innovates the framework system of accident science, and finally realizes the independent discipline system of accident science.

\section{Conclusions}

(1) Accident science can be regarded as a comprehensive science under the intersection of multi-disciplines, and accident science needs the cross-integration of sociology, management, engineering and other disciplines. Various management models can provide theoretical reference and guidance for accident cause models, and sociological methods provide a supporting basis for accident prevention. People are always at the core of social accidents. Realizing the function of the system and ensuring the safety of the system are decisions that people participate in. In accidents, people's cognition and behavior will affect the development and trend of accidents, and the spread and coupling of accidents in the system will also be affected by people. The accident itself has certain system characteristics. An accident is a state in which the various elements of injury are coordinated under a certain morphological structure. The accident is the inevitable result of the increase of system entropy. Entropy is the inherent attribute that leads to the destruction of system balance, and the increase of entropy means that the safety state of the system is challenged.

(2) Safety is an activity carried out in order to enable rational people to achieve a certain intention within a certain scope of time and space, and through the adoption of management and technical means, it achieve the comprehensive state and guarantee conditions of rational people's physical and mental well-being, stability of system elements, control of organizational functions, prevention and resolution of risks. Meanwhile, it explained the connotation of the new security concept and the related concepts. Safety is a comprehensive concept, which can not only reflect the subjective feelings but also depict the objective state. Whether the state is good or not is determined by the internal and external system conditions. If the external conditions can ensure that the people, elements, organizations and risks of the system are under control, then it is called a safety system. The ultimate goal of the new safety is to achieve a normal comprehensive state of the system or to have various conditions under which the state of the system can be guaranteed.

(3) Under a certain category of time and space, accident science is a comprehensive cross-discipline, which taking safety as the purpose and the accident as the research object studies the mechanism and law of accident cause, evolution, disaster or disappearance, accident prediction, prevention optimal control methods engineering measures in the management and technical means of emergency, exploration, identification, treatment and reference of accidents. It is simply understood that accident science is a safety scientific theory that studies the law, management and technology of accidents in the system. The concept of accident science can be summarized as 
"3-4-5" model. That is, the outer layer includes emergency, exploration, identification, treatment, reference and other programs and technical means. The middle layer includes engineering or management measures for the cause, evolution, disaster or disappearance of system accidents. The inner layer includes the prediction, prevention and control methods of an accident.

(4) Accident science is formed by the cross-integration of safety science system, natural science system and management system, which is subordinate to the foundation of safety science. The discipline of safety science has a wide range of research fields and branches, and the accident science as a branch of safety science has a rich discipline system. The main theoretical foundations of accident science are law, psychology, education, behavioral science, management, economics, systems, mathematics, physics, chemistry, biology, logic, investigation, technology and engineering, etc. The main branches of accident science should at least include accident psychology, accident cause theory, accident evolution law, accident prediction and prevention methods, accident emergency management, accident investigation methods and techniques, accident simulation and reproduction technology, accident identification technology and specification, accident control engineering, typical accident cases, accidents in a certain industry, and so on.

(5) The basic theory of accident science is not a self-system with its own profound scientific accumulation, but another system that needs integration and innovation, and constantly absorbs and innovates the methods, principles, procedures and systems of accident science research in the development of the discipline. The research content is the "3-4-5" model, which is divided into the aspects of accident prediction, prevention and control, engineering or management analysis of accident cause, evolution, disaster or disappearance, emergency response, exploration, identification, treatment and reference. To sum up, the main content of accident science research is divided into three parts: accident theory research, accident application management research and basic research of accident science discipline.

\section{Acknowledgments}

I thank anonymous reviewers for critically reading the manuscript and helpful discussions. This research was supported by the Young PhD Teaching and Scientific Research ability Promotion Program of Xi'an Shiyou University (No.134010025).

\section{References}

Adedigba, S.A., Khan, F., Yang, M., 2016. Dynamic safety analysis of process systems using nonlinear and non-sequential accident model. Chemical Engineering Research and Design 111, 169-183.

Allison, R.W., Hon, C.K.H., Xia, B., 2019. Construction accidents in Australia: Evaluating the true costs. Safety Science 120, 886-896.

Amin, S., 2020. Backpropagation - Artificial Neural Network (BP-ANN): Understanding gender characteristics of older driver accidents in West Midlands of United Kingdom. Safety Science 122, 104539.

Aney, M.S., Ho, C., 2019. Deadlier road accidents? Traffic safety regulations and heterogeneous motorists' behavior. Regional Science and Urban Economics 77, 155-171.

Awal, Z.I., Hasegawa, K., 2017. A Study on Accident Theories and Application to Maritime Accidents. Procedia Engineering 194, 298-306.

Benner, L., 2019. Accident investigation data: Users' unrecognized challenges. Safety Science 118, 309-315.

Chen, J., Zheng, H., Wei, L., Wan, Z., Ren, R., Li, J., Li, H., Bian, W., Gao, M., Bai, Y., 2020. Factor diagnosis and future governance of dangerous goods accidents in China's ports. Environmental Pollution 257, 113582.

Dechy, N., Dien, Y., Funnemark, E., Roed-Larsen, S., Stoop, J., Valvisto, T., Arellano, A.L.V., 2012. Results and lessons learned from the ESReDA's Accident Investigation Working Group: Introducing article to "Safety Science" special issue on "Industrial Events Investigation”. Safety Science 50, 1380-1391. 
FENG, Q., 2014. "Tree type" model of accident cause theory. Oil \& Gas Storage and Transportation 33, 115-120+128.

FU, G., SUO, X., JIA, Q., FU, M., 2018. Comparative study on ten accident causation models. Journal of Safety Science and Technology 14, 58-63.

FU, G., WANG, X., LI, Y., 2017. On the 24Model and the application of its causative codes to the analysis of the related accidents. Journal of Safety and Environment 17, 1003-1008.

Fu, G., Xie, X., Jia, Q., Li, Z., Chen, P., Ge, Y., 2020. The development history of accident causation models in the past 100 years: 24Model, a more modern accident causation model. Process Safety and Environmental Protection 134, 47-82.

Fyhri, A., Johansson, O., Bjørnskau, T., 2019. Gender differences in accident risk with e-bikes-Survey data from Norway. Accident Analysis \& Prevention 132, 105248.

GAN, X., CUI, H., GAO, W., DAI, Z., 2015. Analysis and suggestion of midair collision accident based on HFACS. Journal of Safety Science and Technology 11, 96-102.

García-Arroyo, J.A., Osca Segovia, A., 2020. Occupational accidents in immigrant workers in Spain: The complex role of culture. Safety Science 121, 507-515.

Ghandour, A.J., Hammoud, H., Dimassi, M., Krayem, H., Haydar, J., Issa, A., 2020. Allometric scaling of road accidents using social media crowd-sourced data. Physica A: Statistical Mechanics and its Applications 545, 123534.

HENG, W., XU, H., ZENG, C., ZHANG, Y., 2017. Mechanism of Traffic Accidents of Passenger Vehicles Based on the Catastrophe Theory. Safety and Environmental Engineering 24, 141-145.

HUA, L., ZHENG, W., 2019. R esearch on causation of railway accidents based on complex network theory. China Safety Science Journal 29, 114-119.

HUANG, L., WU, C., WANG, B., 2016. Complex chain evolution mechanism and its modeling of major accident based on entropy theory. Journal of Safety Science and Technology 12, 10-15.

Hummel, D.W., Chouhan, S., Lebel, L., Morreale, A.C., 2020. Radiation dose consequences of postulated limiting accidents in small modular reactors to inform emergency planning zone size requirements. Annals of Nuclear Energy 137, 107062.

Joni, H.H., Mohammed, A.A., Shakir, A.A., 2020. Classification of traffic accidents datasets between 2003-2017 in Iraq. Data in Brief 28, 104902.

Jonsson, L., Björklund, G., Isacsson, G., 2019. Marginal costs for railway level crossing accidents in Sweden. Transport Policy 83, 68-79.

Kumar, P., Gupta, S., Gunda, Y.R., 2020. Estimation of human error rate in underground coal mines through retrospective analysis of mining accident reports and some error reduction strategies. Safety Science 123, 104555.

Landay, K., Wood, D., Harms, P.D., Ferrell, B., Nambisan, S., 2020. Relationships between personality facets and accident involvement among truck drivers. Journal of Research in Personality 84, 103889.

LI, X., LI, H., LIU, C., 2019. Hazard Area Model of Accumulation and Amplification Effect for Hazardous Chemical Coupling Accidents. Industrial Safety and Environmental Protection 45, 37-40.

LIN, Y., 2019. Analysis of the public opinion on the Internet about the coal mine safety accidents based on dynamic thematic emotion model. Journal of Safety and Environment 19, 1288-1295.

LIU, Q., 2010. Establishment and Practice of Safety Science and Discipline Chemical Industry, Beijing.

Liu, Q., Liu, J., Gao, J., Wang, J., Han, J., 2020. An empirical study of early warning model on the number of coal mine accidents in China. Safety Science 123, 104559.

LUO, T., WU, C., 2018. Discussion on the fundamental issues about accident. Journal of Catastrophology 33, 5-12.

Luo, T., Wu, C., 2019. Safety information cognition: A new methodology of safety science in urgent need to be established. Journal of Cleaner Production 209, 1182-1194.

Mauro, J.C., Diehl, B., Marcellin, R.F., Vaughn, D.J., 2018. Workplace accidents and self-organized criticality. Physica A: Statistical Mechanics and its Applications 506, 284-289.

Mokarami, H., Alizadeh, S.S., Rahimi Pordanjani, T., Varmazyar, S., 2019. The relationship between organizational safety culture and unsafe behaviors, and accidents among public transport bus drivers using structural equation modeling. Transportation Research 
Part F: Traffic Psychology and Behaviour 65, 46-55.

Naghavi K, Z., Mortazavi, S.B., Asilian M, H., Hajizadeh, E., 2019. Exploring the Contributory Factors of Confined Space Accidents Using Accident Investigation Reports and Semistructured Interviews. Safety and Health at Work 10, 305-313.

Naqvi, N.K., Quddus, M.A., Enoch, M.P., 2020. Do higher fuel prices help reduce road traffic accidents? Accident Analysis \& Prevention 135, 105353.

Nishijima, M., Rocha, F.F., 2020. An economic investigation of the dengue incidence as a result of a tailings dam accident in Brazil. Journal of Environmental Management 253, 109748.

Oettingen, M., 2020. A criticality study on the LA-1 accident using Monte Carlo methods. Nuclear Engineering and Design 359 , 110467.

Parsa, A.B., Movahedi, A., Taghipour, H., Derrible, S., Mohammadian, A., 2020. Toward safer highways, application of XGBoost and SHAP for real-time accident detection and feature analysis. Accident Analysis \& Prevention 136, 105405.

Tongyuan, L., WU, C., 2018. Knowledge and Strategy on the Basic Problems of Accidents. Journal of Catastrophology 33, 5-12.

TU, S., JIA, M., WU, C., WANG, B., 2018. Study on occurrence mechanism and its models of malignant human accidents. Journal of Safety Science and Technology 14, 180-187.

Underwood, P., Waterson, P., 2013. Systemic accident analysis: Examining the gap between research and practice. Accident Analysis \& Prevention 55, 154-164.

WANG, M., CHEN, P., CHEN, Z., WEI, Y., XU, Z., 2019. Analysis Method of Accidents in Geophysical Prospecting Operation Based on Text Mining. Journal of Xi'an Shiyou University(Natural Science Edition) 34, 119-126.

WU, C., 2007. Initial study of the science of safety science. China Safety Science Journal 17, 5-15.

XIAO, Z., WANG, X., ZHANG, W., 2017. Analysis for the ship grounding accidents based on the Bayesian network model. Journal of Safety and Environment 17, 418-421.

Youkhana, B., Tavassol, F., Johannsen, H., Spalthoff, S., Gellrich, N.-C., Stier, R., 2019. An in-depth technical and medical investigation of facial injuries caused by car accidents. Injury 50, 1433-1439.

YU, F., SONG, Y., MENG, F., FANG, D., 2016. Mechanism and risk evaluation of pedestrian stampede accidents in public places of cities-A level influence model based on the event system theory ( EST). Science Research Management 37, 162-169.

Zaranezhad, A., Asilian Mahabadi, H., Dehghani, M.R., 2019. Development of prediction models for repair and maintenance-related accidents at oil refineries using artificial neural network, fuzzy system, genetic algorithm, and ant colony optimization algorithm. Process Safety and Environmental Protection 131, 331-348.

ZHANG, J., LUO, C., CHENG, M., ZHAO, Y., 2019a. Structural equation model between safety accidents and organizational culture of construction enterprises. China Safety Science Journal 29, 129-136.

Zhang, J., Xu, K., Reniers, G., You, G., 2020. Statistical analysis the characteristics of extraordinarily severe coal mine accidents (ESCMAs) in China from 1950 to 2018. Process Safety and Environmental Protection 133, 332-340.

ZHANG, L., LIU, T., PAN, F., GUO, T., LIU, R., 2014. Analysis of effects of driver factors on road traffic accident indexes. China Safety Science Journal 24, 79-84.

Zhang, M., Zheng, F., Chen, F., Pan, W., Mo, S., 2019b. Propagation probability of domino effect based on analysis of accident chain in storage tank area. Journal of Loss Prevention in the Process Industries 62, 103962.

ZHANG, Y., 2013. Analysis on the treatment and prevention mechanism of college students' injury accidents. Modern Reading 06, 43.

ZHOU, X., GUO, S., 2019. Mechanism of Behavioral Risk Chain for Building Construction Accident Journal of Engineering Management 33, 112-116. 